\title{
Methodology of design of wind turbine tower structures
}

\author{
Avelino Alves Filho \\ Lincoln Sadao Mazakina China
}

\begin{abstract}
The present work analyzes the methodology of design of wind turbine tower structures considering the dynamical loads obtained using a spectral formulation for the time distribution of wind velocities. The time varying loads thus obtained comprise those due to the wind action on the tower and those due to wind imposed loads on the nacelle and rotor blades. The analysis comprised the calculation of the tower structure response using the shell element and the beam element methodologies. Stress values obtained with the beam element are then compared with values obtained using the shell element model that takes into account the local stress concentrations. Since the basic objective is the global dynamic response of the structure, beam element models provide an efficient and rapid way to obtain the desired dynamic response. Linear dynamical analyses were obtained using a modal superposition technique and compared to a nonlinear analysis obtained using a direct integration technique. Results of dynamical analysis obtained using the beam element model show good agreement with results using the shell element model, enabling a more rapid and efficient methodology to be used in conjunction with the spectral formulation.
\end{abstract}

\section{Introduction}

The objective of this work is to obtain the dynamic response of a tower using the resources of Finite Element Method. It was performed by two procedures, Linear Analysis by Modal Superposition Method, that is interesting for linear behavior of the structure, and in complementation Direct Integration, that is recommended for nonlinear behavior of the structure. Initially, we introduce some considerations about the basis of two Methods, and after, we present the results for both analyses. The excitations that are considered in the analysis were obtained by spectral formulations that represent excitations from the wind action and the effects of mechanical devices installed at the tower. Some comments about this spectral formulations and excitations forces are made after, but, the central objective of this work is the structural response of a tower by different methods, and two different types of finite element models, since that the Global dynamic response is the main objective. In this line of action, is important to choice the Beam Element model for global dynamic analysis and after, stablishes correlations with thin shell element model. The Dynamic response by Beam Elements for Global
Behavior is very efficient, because with a small number of Differential Equations that takes part of a system, the numerical solution has a fast solution, against the time that would be spent for a thin shell dynamic model with some millions of differential equations that would be solved by discrete methods

\section{Fundamentals for solve dynamics responses using both methods}

\section{a. Modal Method}

The great number of differential equations that represents the dynamic behavior of several nodes of Finite Element Model of tower structure can be represented in compact form by matrix notation:

$$
[M] \cdot\{\ddot{U}\}+[C] \cdot\{\dot{U}\}+[K] \cdot\{U\}=\left\{F_{(t)}\right\}
$$

In mathematic point of view, this is a System of Second Order Differential Equations. These equations are not independent. They are connected and to solve the complete system is not a sample problem. One of the methods that permits to solve this assembly of equations in more sample way is to disconnect the assembly of equations by the concept of modal coordinates and obtain the dynamic response by the sum of vibrations modes. In this kind of strategy, each mode contributes with a modal participation factor. These modal participations factor is solved from orthogonality properties. The eigenvectors are orthogonal in relation to Stiffness Matrix, Mass Matrix, Damping Matrix. From application of these properties we can solve for each mode the modal mass, modal damping, modal stiffness and modal force. So, we can obtain the Tower Response Dynamic by the sum of the several modes elected to represent the dynamic characteristic of structure. Each vibration mode is multiplied by a coefficient that represents the importance of this mode in the Structural Response, or, the weight or participation factor of this mode in the response analysis. 
Proc. of the Third Intl. Conf. on Advances in Civil, Structural and Construction Engineering - CSCE 2015

Copyright (C) Institute of Research Engineers and Doctors, USA .All rights reserved.

ISBN: 978-1-63248-079-8 doi: 10.15224/ 978-1-63248-079-8-74

These weights related to each mode are a function of time, and in each time instant we have a different structural response. This idea is represented in (2):

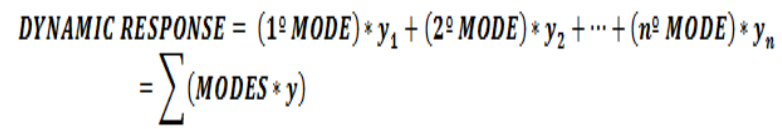

Then, to obtain the Dynamic Response of the Tower Structure, we need to solve two steps.

- First, is the calculation of Structural Mode shapes and Natural Frequencies of the Tower, the Modal Analysis

- Second, the determination of Participation Factor related to each mode of structure vibration.

Then, based in Modal Superposition Method we have

$\{U(t)\}=\left\{\begin{array}{c}U_{1}(t) \\ U_{2}(t) \\ U_{3}(t) \\ U_{4}(t) \\ \vdots \\ \vdots\end{array}\right\}=y_{1} \cdot\left\{\begin{array}{c}U_{11} \\ U_{21} \\ U_{31} \\ U_{41} \\ \vdots \\ \vdots\end{array}\right\}+y_{2} \cdot\left\{\begin{array}{c}U_{12} \\ U_{22} \\ U_{32} \\ U_{42} \\ \vdots \\ \vdots\end{array}\right\}+y_{3} \cdot\left\{\begin{array}{c}U_{13} \\ U_{23} \\ U_{33} \\ U_{43} \\ \vdots \\ \vdots\end{array}\right\}+\cdots \cdots \cdots+y_{n} \cdot\left\{\begin{array}{c}U_{1 n} \\ U_{2 n} \\ U_{3 n} \\ U_{4 n} \\ \vdots \\ \vdots\end{array}\right\}$

$=\quad \sum_{i=1}^{n} y_{i}(t) \cdot \phi_{i}$

or

$$
\begin{aligned}
& \{U(t)\}=y_{1} \cdot\left\{\phi_{1}\right\}+y_{2} \cdot\left\{\phi_{2}\right\}+y_{3} \cdot\left\{\phi_{3}\right\}+\cdots \cdots \cdots \cdots+y_{n} \cdot\left\{\phi_{n}\right\} \\
= & \sum_{i=1}^{n} y_{i}(t) \cdot\left\{\phi_{i}\right\}
\end{aligned}
$$
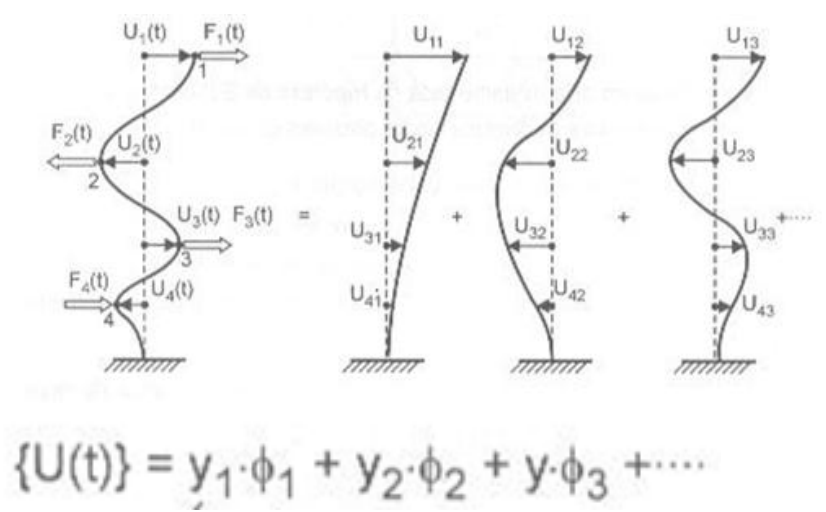

Figure 1. Modal Superposition to Linear Systems.

By substitution the (4) in (1) and developing the derivatives we have:
$[M] \ddot{y}_{1} \cdot\left\{\phi_{1}\right\}+[M] \ddot{y}_{2} \cdot\left\{\phi_{2}\right\}+[M] \ddot{y}_{3} \cdot\left\{\phi_{3}\right\}+\cdots \cdots \cdots+[M] \ddot{y}_{n} \cdot\left\{\phi_{n}\right\}+[C] \dot{y}_{1} \cdot\left\{\phi_{1}\right\}+[C] \dot{y}_{2} \cdot\left\{\phi_{2}\right\}+[C] \dot{y}_{3} \cdot\left\{\phi_{3}\right\}+\cdots \cdots$ $+[C] \dot{y}_{n} \cdot\left\{\phi_{n}\right\}+[K] y_{1} \cdot\left\{\phi_{1}\right\}+[K] y_{2} \cdot\left\{\phi_{2}\right\}+[K] y_{3} \cdot\left\{\phi_{3}\right\}+\cdots \cdots \cdots+[K] y_{n} \cdot\left\{\phi_{n}\right\}=F(t)$ (5)

The (6) can be simplified by the use of Orthogonality Property, that is applied to the Stiffness Matrix, Mass Matrix, Damping Matrix. It is important to mention that Damping Matrix defined on the proper mode by Rayleigh relation, also obey the Orthogonality Property. It is made by the Proportional Damping, as defined in reference (1). Then:

$$
\begin{gathered}
\left\{\phi_{j}\right\}^{T} \cdot[K] \cdot\left\{\phi_{i}\right\}=0 \\
\left\{\phi_{j}\right\}^{T} \cdot[M] \cdot\left\{\phi_{i}\right\}=0 \\
\left\{\phi_{j}\right\}^{T} \cdot[C] \cdot\left\{\phi_{i}\right\}=0
\end{gathered}
$$

with $i \neq j$

If at the equation (5) both members are pre multiplied by the same eigenvector $\left\{\phi_{i}\right\}^{T}$, in all terms where $\mathrm{i} \neq \mathrm{j}$ the product will be zero. For de same eigenvector the product will be:

$k_{i}=\left\{\phi_{i}\right\}^{T} \cdot[K] \cdot\left\{\phi_{i}\right\}$ - modal Stiffness for the mode i

$m_{i}=\left\{\phi_{i}\right\}^{T} \cdot[M] \cdot\left\{\phi_{i}\right\}$ - modal mass for the mode i

$c_{i}=\left\{\phi_{i}\right\}^{T} \cdot[C] \cdot\left\{\phi_{i}\right\}$ - modal damping for the mode $\mathrm{i}$

$f_{i}(t)=\left\{\phi_{i}\right\}^{T} \cdot\{F(t)\}$ - modal force for the mode i

That is the (8).

The factor of participation of each mode in the response analysis can be obtained by similar way. When we perform the modal analysis, for each mode we have the correspondent values obtained by (8) and for each mode we have only to solve a scalar equation for resolve the factor of participation of each mode. Then:

$$
m_{i} \cdot \ddot{y}_{i}(t)+c_{i} \cdot \dot{y}_{i}(t)+k_{i} \cdot y_{i}(t)=f_{i}(t)_{(9)}
$$

The (9) is the famous equation of One Degree of Freedom System. In this equation the coordinate $y_{i}$ is not the physical coordinate of the structural model in 3D space. The solution of a sample One Degree of Freedom System gives us the participation factor of each mode in the response of the tower. These coordinates are named as Modal Coordinates, Generalized Coordinates or Normal Coordinates.

The figures (2) and (3) give a Graphical and summary idea of the concepts that will be applied to obtain the response dynamic of the tower 


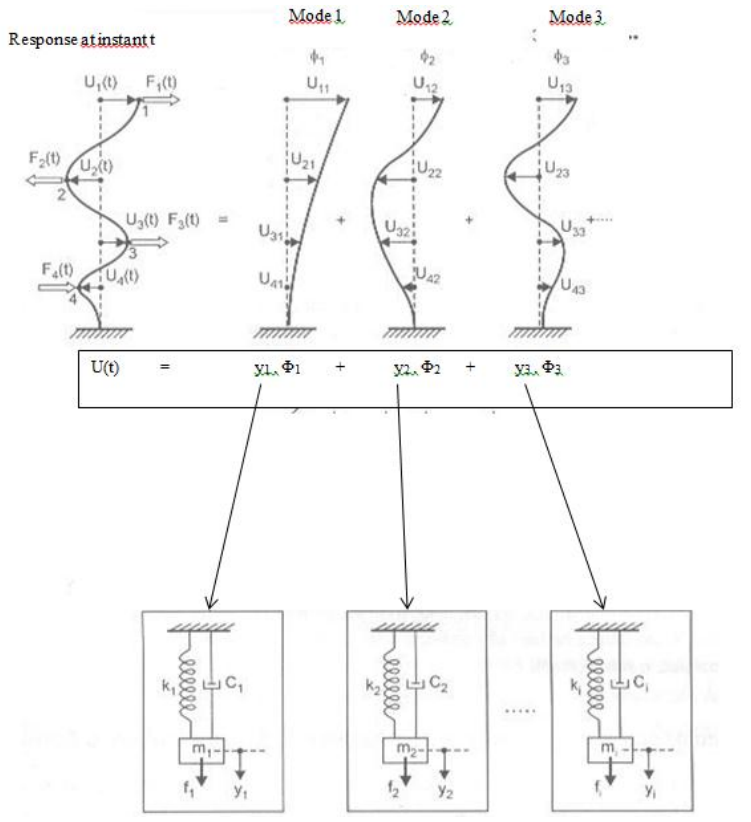

Figure 2. Modal system associate to each mode of vibration. The dynamic response is obtained by sum of modes. The modal participation of each mode in response is obtained by the solution of One Degree of Freedom System associate to each mode.

By Finite Element Method determine the
eigenvalues and eigenvector $\left(\lambda_{\mathrm{i}}, \varnothing_{\mathrm{i}}\right)$

Modal Stiffness for the mode $i$, modal mass for the mode $i$, modal damping for the mode $i$, modal force for the mode $i$. For each mode determine modal properties

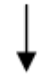

Solve the modal system for each mode and then determine $y_{i}$

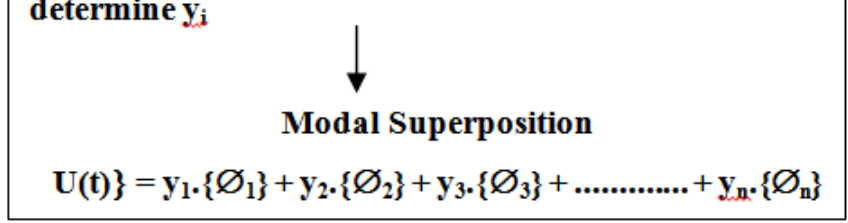

Figure 3. General steps for Modal Superposition to obtain the Response Structural Dynamics of the Tower.

\section{b. Direct Integration Method}

In the Method of Direct Integration the Assembly of differential equations is submitted a numerical integration step-by-step. This means that in opposite form of the Modal Superposition, no transformation in the system of differential equations is performed before the integration process is developed. Normally, this method is adequate for nonlinear behavior of the structure, for example, when the displacements are large. The consequence is that the stiffness change during the time variation, and the modal analysis would be not recommended.

Two points are important to mentions in direct integrations schemes:

a) The dynamic behavior of several nodes of Finite Element Model of tower structure and represented in compact form by matrix notation is satisfied only in some discrete instants of time, in the interval that the dynamic response is performed. The total time of the analysis is divided in time steps. The choice of time steps in Direct Integration has a procedure defined in reference (2)

b) Inside each time interval during the total time of analysis, between two consecutives instants of time, a law for displacement, velocities and accelerations is assumed.

The definition of Integration Direct methods, such as Explicit and Implicit, can be read at reference (2). The basis for develop the Algorithms is the (1) and the Taylor's Series, accurate of second order. In the development of Taylor's Series formulations, the derivatives until second order have physical meaning. They are Accelerations, Velocities and Displacements of the Nodes of Structural Model, or more properly, these Physical quantities for Degree of Freedom.

All details of the development of Direct Integrations are presented in reference (2)

\section{Finite Elements Models}

Before we present the solution of Dynamic response by Finite Element Method, it is important to discuss two types of Models that were used for Project Development.

Two type of response is interesting for the tower. One is the response of structure for Peak Loads, for example, the loads caused by the wind, for maximum condition. Another is the response under action of variable loads from the wind that can be obtained from spectral formulation. The Peak Load represent the maximum solicitation at the top of the Tower. For example for the case that we will present as a case, the tower has a height of $90 \mathrm{~m}$. The horizontal force generated by the wind maximum action is near $1.000 .000 \mathrm{~N}$. This value of force is determined by spectral formulation and normative loads that is applied for this analysis. Normally this peak load is considered to verify the behavior of structure under de yield conditions. The von Mises Criterion, is based in maximum octahedric Shear stresses that is verified in the faces of regular octahedral that has equals angles with the principal stress axes direction neighborhood a structure point.

Another structural response is the behavior of the tower under the variable loads caused by wind actions and that can be obtained from spectral formulation that will be commented after. Other variable loads are also applied in the structure, caused by several devices installed at the top of the tower. This is a Dynamic Analysis. With the results of Dynamic Analysis we can perform fatigue analysis in all points of structure. Some these points are in conditions of "parent material", that the fatigue limit can be defined in first instance from the ultimate strength of the steel, considered in this application. In other points, we have welded joints, and for them we need consider the reduction of fatigue limit for this application. We use in that case the "material independent joint factors", associated to quality process of welding and the type of welded joint.

In Dynamic point of view, the great objective is to determine the dynamic amplification of the tower. As we will observe after, the tower together the great masses that is 
added to the structure, provides values of natural frequencies very low.

In other words, the main objective in dynamic point of view is to determine the primary structural response of the tower. From these considerations it is important to consider the possibility to construct two different types of Finite Element Models. The Beam Element Model of the tower with lumped mass that represents que devices installed at the tower, and the thin shell element models that permits to obtain stresses with the respective stress concentrations factor in all joints.

It is possible with a static analysis to correlate the primary stresses obtained by both models. It is very economic analysis obtains stresses from models constructed by beams elements. The question is that the global dynamic response for primary behavior of tower can be obtained from Beam model that has a small numbers of degree of freedom. We can verify this condition when we compare the natural frequencies obtained from thin shell models and beams models. The global dynamic primary behavior of the tower that is expressed by modal analysis are the same. Modal analysis reflect as the structure will respond to the dynamic loads.

It is important to mention that in stage of $R \& D$ project, this approach to obtain primary behavior of structure, is the key point, and can made by economic process. Thin shell models with millions of degree of freedom for Global Dynamic Analysis can be Impractical.

The stresses in details of the tower, as bolts joints, welded joints, local reinforcement can be determined by thin shell models, using the stresses that would be corrected from the global dynamic analysis from beams models. Then, beams models can help us to solve the dynamic global response and thin shell models, in the next stage of project, help us do determine the stresses with all concentrations factors that is function of several details of the tower.

In the figure 4 is represented the Finite Element Model with Thin Shell Elements and also in Beam Elements. The model by thin shell elements considers by regions different physical properties associated to the thickness that change for each region of the tower that has a conical form. The mesh was generated at midsurface by region. The thickness is a function of the tower height. By the other hand, the beam model is generated over the midline of the sections of the conical tower, and the physical properties are defined using the Beam Element, by the beam sections.

Both models were constructed in preprocessing resources of FEMAP, version 10.3. The Solver was the NX Nastran that works together Femap.

When we use the resource of shaded representation the beam element appear as a "shell", but only for proposes of visual representation, because the beam theory, as well be known, consider the midline of the beam in the neutral axis of the section.

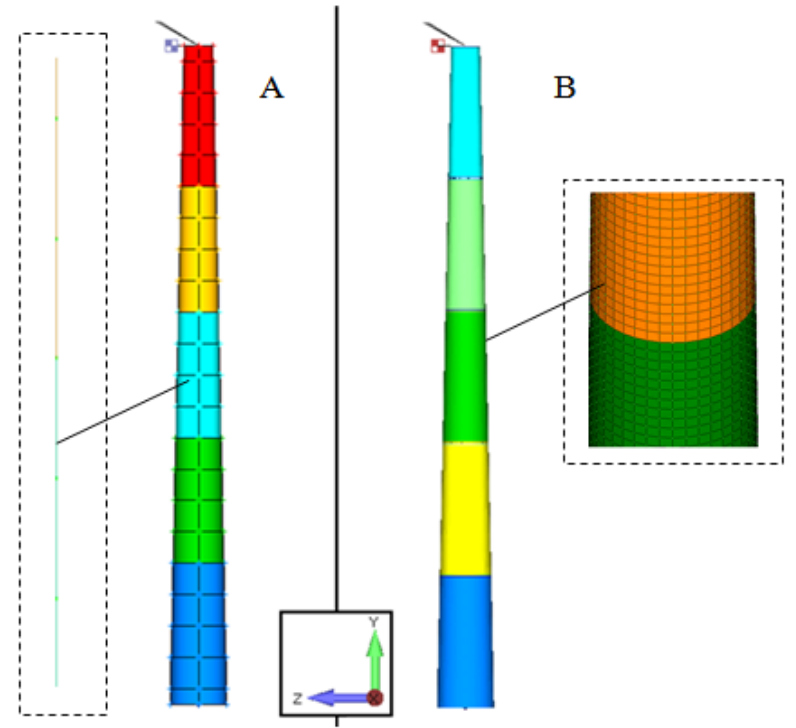

Figure 4. Finite Element Model of the Tower with Beam Elements in A, and Finite Element Model with Shell Elements in B.

\section{Loads}

The main objective of this work, is to compare the Dynamic Primary Global Response of the Tower, by economic model using beam elements for determine the dynamic amplification in Global Response, as an alternative to thin shell models of overall model. The last mentioned shell model would be very expensive in function of the great number of degree of freedom. In particular, in R\&D phase of a new project, this approach would be convenient. It is a "sample", economical and fast procedure to solve dynamic response for beams models, and in R\&D phase we can reproduce several finite element models to gain a sensitive analysis or several alternative of projects.

Then, we will give the central focus to the wind loads in this work to discuss the Methodology, because the procedure for solve dynamic analysis for General Dynamic Load, is a well-known technic using Duhamel's Convolution Integral. Then, the procedure can be applied for all other dynamics loads present in the project.

The average values obtained by meteorological sensors are in general, to a time interval of 10 minutes. The values thus obtained are a sample of the winds of the region.

The Representation of the distribution of wind speeds is made by a probability density function called Weibull distribution.

The method of verisimilitude is used for the determination of the form factor, essential for the calculation of the energy produced. Through the distribution of Weibull is calculated over a time interval equal to $10 \mathrm{~min}$ average of horizontal speed component.

The fluctuations of speeds around the average value in a time interval $\Delta \mathrm{T}$ characterize the turbulence of wind regime and its "variability" during the interval of observation $\mathrm{T}$.

This "variability" is a function of the time interval considered. The variation of wind speed for the various sampling intervals (month, day, hours, minutes, seconds) has been shaped by Van der Hoven. 
This modeling considered the wind speed as a random variable continues with a spectrum of energy constituted basically by three peaks.

The fluctuations of velocities $\mathrm{u}(\mathrm{z} ; \mathrm{t})$ and of interest to characterize the dynamic response of structures correspond to third peak of the spectrum of Van der Hoven. This region of the spectrum can usually be characterized by spectral formulations of broad acceptance in structures dynamics. The formulations in use are of Kaimal and Von Karman formulations

It is worth mentioning that the information presented here constitute only a reference of as was performed the study of winds that are input data of structural analysis, and developed by the corresponding project team. In the figure (5) we represent the wind velocities obtained from this project team.

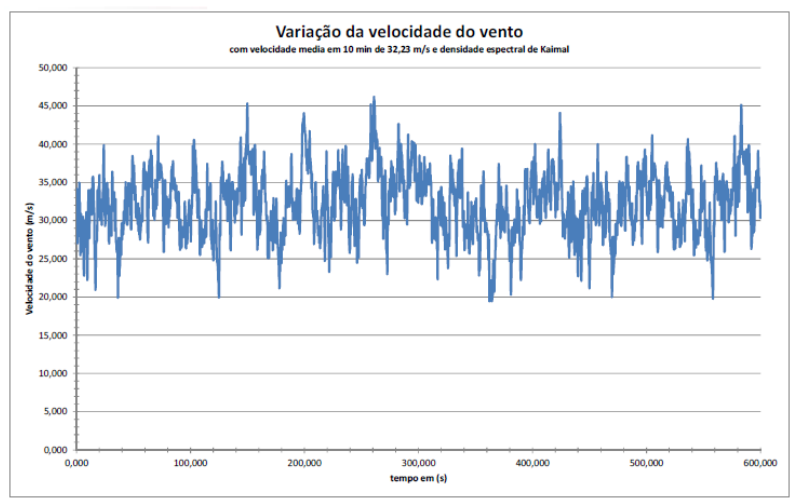

Figure 5. Wind Velocities Variation in $\mathrm{m} / \mathrm{s}$

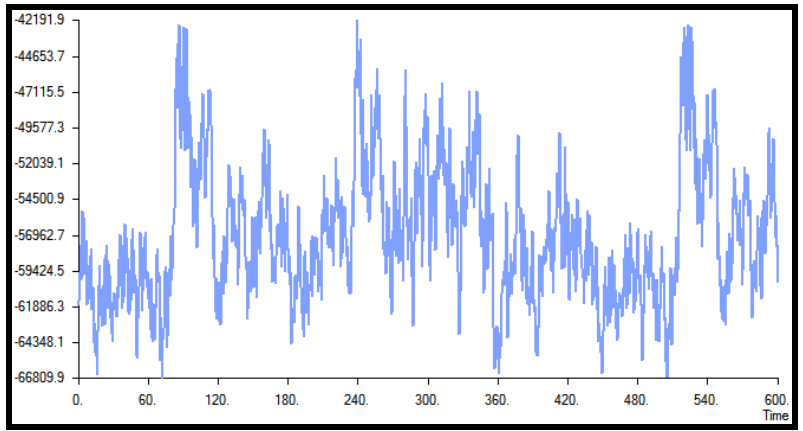

Figure 6. Rotor Temporal Wind Spectre. The Forces are in Kgf or N/9.8.

In the next figure, the figure 7 , is represented the wind forces in several locations of the tower, in two horizontal perpendicular directions. In NX Nastran with Femap representation the first number is a unitary load that is multiplied by a number inside parenthesis. This last number is a function that represents in each location of the tower the wind forces calculated by wind formulations that we mentioned before.

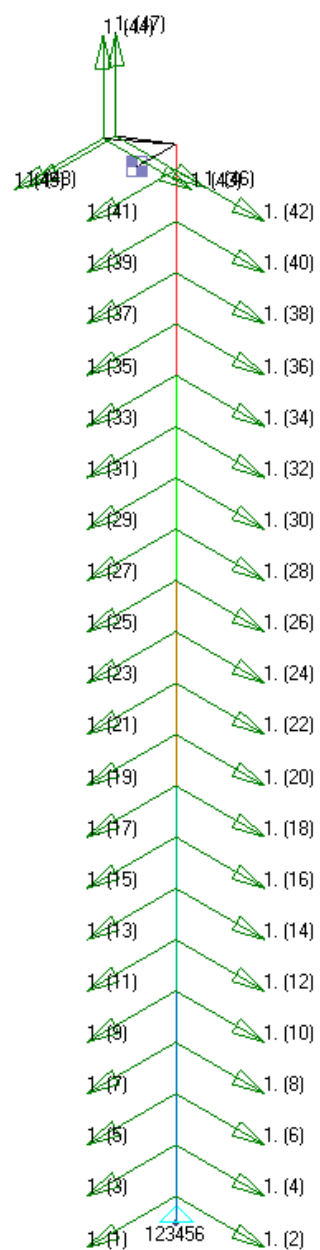

Figure 7. Wind load forces in several locations of the tower, using the formulations mentioned for wind.

\section{v. Dynamic Response of the Tower under Wind Loads}

After all considerations that we have placed before, we will solve the Dynamic Response Analysis of the tower, considering the Modal Superposition Method, based in modal Analysis, and after, The Direct Integration Method, and perform a comparative vison of both methods and results.

\section{a. Dynamic Response by Modal Method}

In this analysis process there is a central point that is very important to consider in initial stages of $R \& D$ Projects and also in other stages of project, that is the use of finite element models based in beams elements for global structural tower behavior .

We developed modal analysis in both finite element models - beams and shells - and observed the results for natural frequencies. It is very important to compare these results that are shown in the Table I.

The natural frequencies solved by Beam Element Model and Thin Shel Model that refers a global vibration modes, gives values very consistent. 
Proc. of the Third Intl. Conf. on Advances in Civil, Structural and Construction Engineering - CSCE 2015

Copyright (C) Institute of Research Engineers and Doctors, USA .All rights reserved.

ISBN: 978-1-63248-079-8 doi: 10.15224/ 978-1-63248-079-8-74

TABLE 1. THE NATURAL MODES AND FREQEUNCIES

\begin{tabular}{|c|c|c|}
\hline $\begin{array}{c}\text { Mode of Natural } \\
\text { Vibration }\end{array}$ & $\begin{array}{c}\text { BEAM Element } \\
\text { Model }\end{array}$ & $\begin{array}{c}\text { Thin Shell Element } \\
\text { Model }\end{array}$ \\
\hline \hline 1 & 0.45 & 0.44 \\
\hline 2 & 3.03 & 2.98 \\
\hline 3 & 6.65 & 6.5 \\
\hline
\end{tabular}

The natural frequencies solved by Beam Element Model and Thin Shell Model that refers a global vibration modes, gives values very consistent. The frequencies of vibrations associated to eigenvalues and eigenvectors are practically equals. Then, the global response of the tower, and the consequent dynamic amplification for the global modes are the same when we use one or other model.

This is a very important conclusion that gives a confidence in use of Beam Element Models for solve Dynamic Response of Global Behavior of the Tower, and preview some possible resonances in the structure.

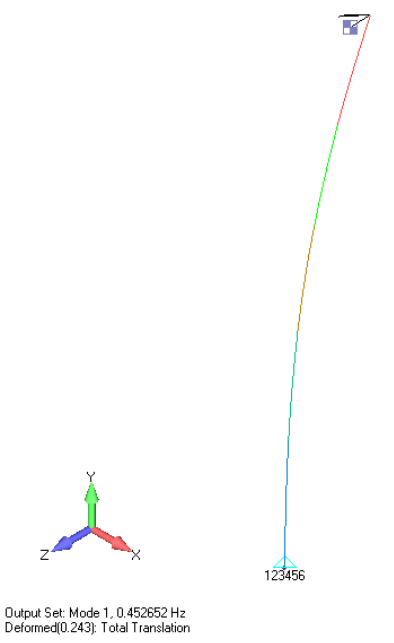

Figure 8. BEAM Element Model - First Natural Mode of Vibration. First Mode Natural frequency is $0.45 \mathrm{~Hz}$.

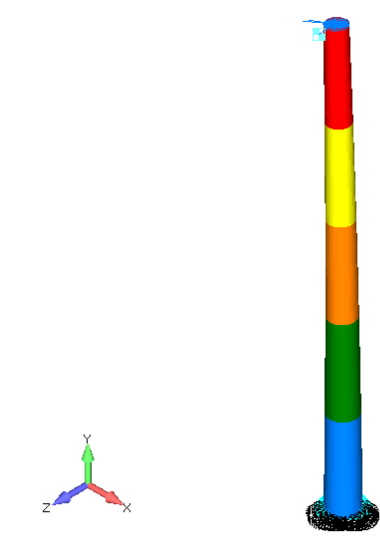

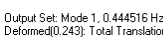

Figure 9. Thin Shell Element Model - First Natural Mode of Vibration. First Mode Natural frequency is $0.44 \mathrm{~Hz}$. Both models have the same natural frequency.

The figure 10 represents the maximum axial bending stresses variations along time for a point in basis of the tower. During the initial instant we have the transitory effect that along time disappear by damping action, and the structure is under permanent general dynamic load. The Maximum stress is $130.5 \mathrm{MPa}$ for transitory stress at the start of analysis.

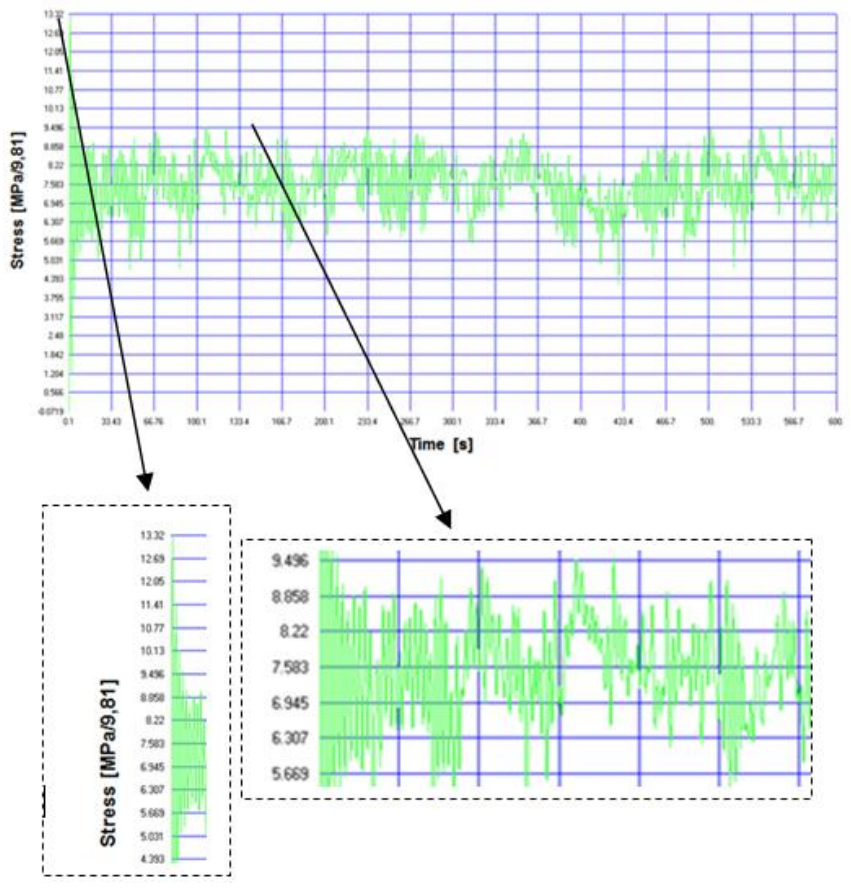

Figure 10. Stress response in a point of maximum stress in the basis of tower.

The parameters that was used as a input to perform Dynamic Analysis by Modal Method is presented as a reference in the figure 11 .

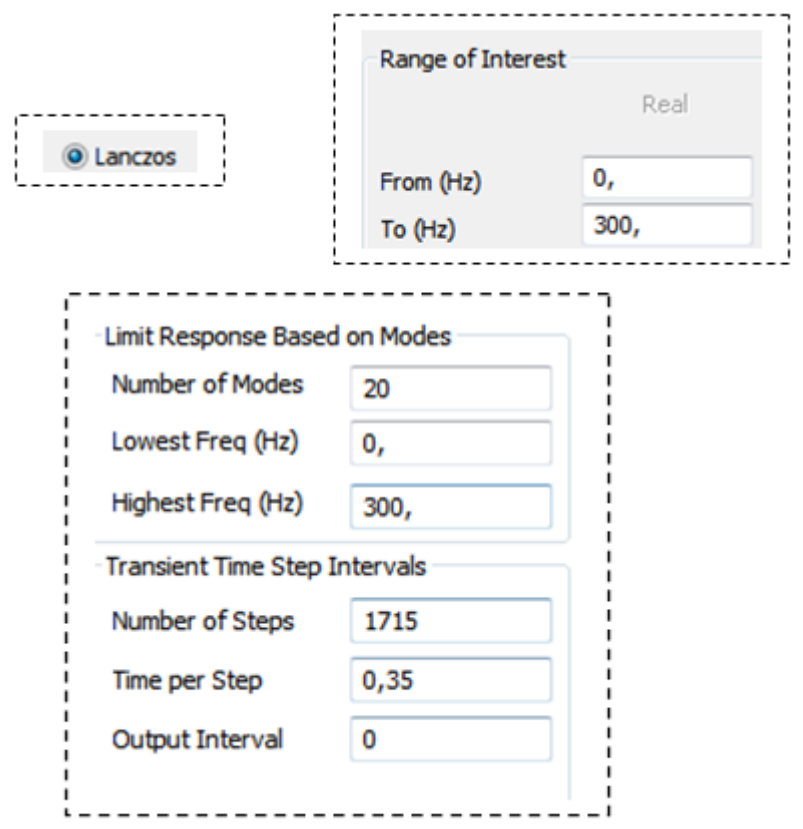

Figure 11. Parameters for Dynamic Analysis by Modal Superposition. 
Proc. of the Third Intl. Conf. on Advances in Civil, Structural and Construction Engineering - CSCE 2015

Copyright (C) Institute of Research Engineers and Doctors, USA .All rights reserved.

ISBN: 978-1-63248-079-8 doi: 10.15224/ 978-1-63248-079-8-74

\section{b. Dynamic Response by Direct Integration}

The other procedure that was used for solve for Dynamic Response is the Direct Method, as mentioned before. Some considerations only to take as a reference, in the same presentation that we observe in the item of Modal Superposition, we can observe for the Direct Method.

In accordance with the manual "NX Nastran Basic Dynamic Analysis User's Guide, we have:

- $\mathrm{G}=$ overall structural damping coefficient (PARAM,G)

- $\quad \mathrm{W} 3=$ frequency of interest in radians per unit time (PARAM,W3), is the conversion of overall structural damping into equivalent viscous damping

- Two parameters are used to convert structural damping to equivalent viscous damping. An overall structural damping coefficient can be applied to the entire system stiffness matrix using PARAM,W3,r where $r$ is the circular frequency at which damping is to be made equivalent. This parameter is used in conjunction with PARAM,G. The default value for $\mathrm{W} 3$ is 0.0 , which causes the damping related to this source to be ignored in transient analysis.

- It was considered as the parameter W3 the value of the first natural frequency of TOWER $(0.33 \mathrm{~Hz})$.

The parameters that was used as a input to perform Dynamic Analysis by Direct Integration is presented as a reference in the figure 12
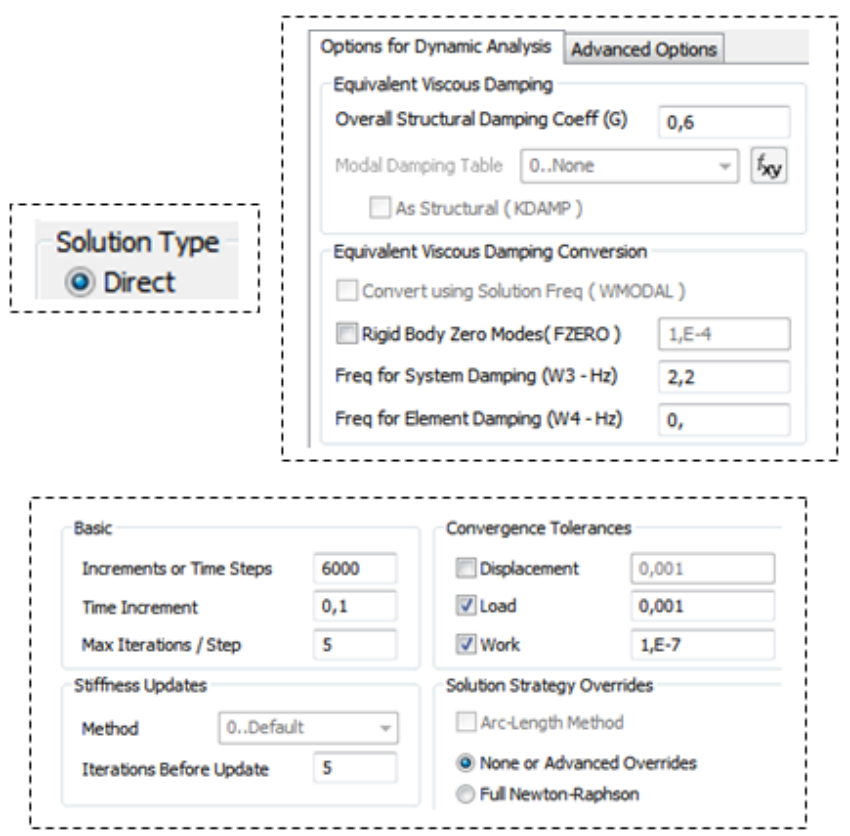

Figure 12. Parameters for Dynamic Analysis by Direct Integration.

The figure 13 represents the maximum axial bending stresses variations along time for a point in basis of the tower, but in this case determined by direct integrations in opposite form to modal method. During the initial instant we have the transitory effect that along time disappear by damping action as we observed in modal method, and the structure is under permanent general dynamic load. The Maximum stress is $139.3 \mathrm{MPa}$ for transitory stress at the start of analysis.

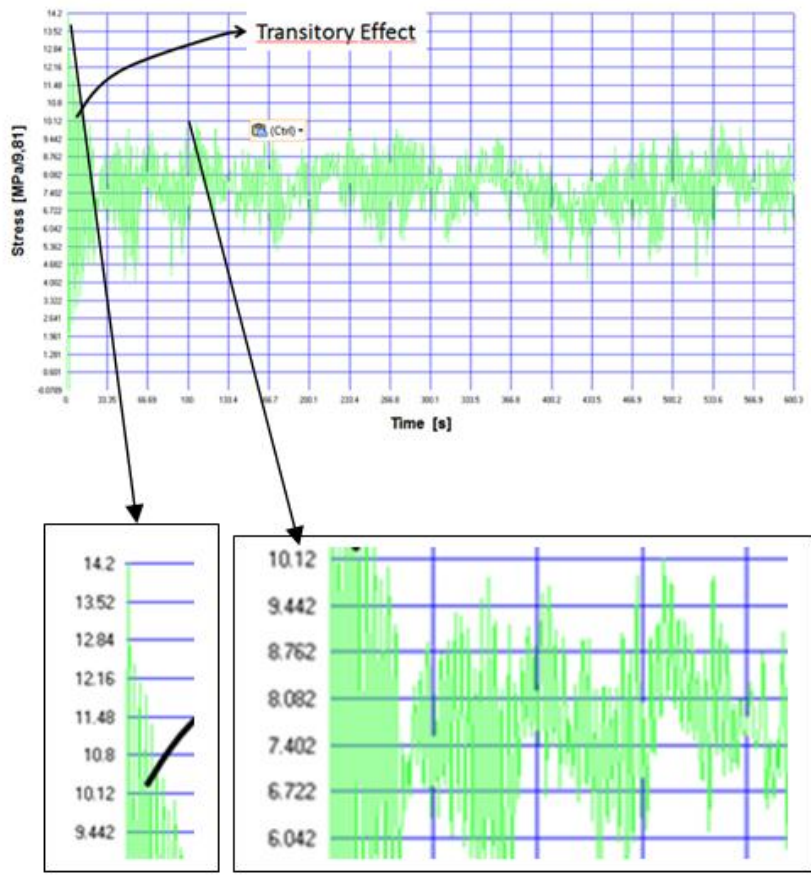

Figure 13. Stress response in a point of maximum stress in the basis of tower.

The difference between linear and nonlinear analysis for stresses solution is $6.74 \%$ in the peak in the start of excitations represented by transitory effect, and in the permanent response for General Dynamic Load the difference is $6,57 \%$.

Of course, in terms of Dynamic Analysis this conditions are applied for this particular problem. In all general cases, the Dynamic response should be calculated for each particular Dynamic characteristics of the structure that is object of analysis. But, the Methodology present in this work is the same and could be used for all applications. When we have displacement that are out of the hypothesis of small displacements, the Direct Integration Method should be used.

\section{vI. Conclusions}

The objective of this work was to introduce a strategy of Analysis to understand the dynamic behavior of wind turbine tower structures. The main focus is to present a Methodology that can be used to perform dynamic analysis. The overall dimensional details of the project that motivate this work, was not presented in this paper. The methods and results obtained would be a guide to develop this kind of analysis. The most important point is to define the use of different types of models, and, with a sample beam element model, the dynamic primary behavior of tower can be solved. The relations between different models can be performed in the sample way. The important point is that the global dynamic amplifications of the tower under the dynamic loads, are the same in the Thin Shell Element Model and Beam Model, and resonance could be analyzed by sample and fast procedure. 
Proc. of the Third Intl. Conf. on Advances in Civil, Structural and Construction Engineering - CSCE 2015

Copyright (C) Institute of Research Engineers and Doctors, USA .All rights reserved.

ISBN: 978-1-63248-079-8 doi: 10.15224/ 978-1-63248-079-8-74

\section{Acknowledgment}

The authors would like thank the following entities:

\section{AES BRASIL}

FUNDEB - The foundation for the development of the City of Bauru - Sao Paulo State - Brazil

\section{References}

[1] A. Filho. Avelino, "Elementos Finitos - A Base da Tecnologia CAE

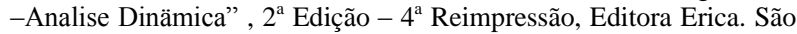
Paulo, Brazil, 2008.

[2] A. Filho. Avelino, "Elementos Finitos - A Base da Tecnologia CAE -Analise não Linear" , $1^{\text {a }}$ Edição, Editora Erica. São Paulo, Brazil, 2012.

[3] A. Filho. Avelino, "Elementos Finitos - A Base da Tecnologia CAE,

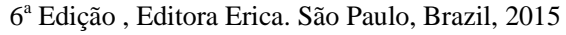

[4] Clough, R.W.; Penzien, J. Dynamics of Structures. New York: McGraw-Hill Book Company,1975

[5] Zienkiewicz,O. C. The Finite Elementt Method, 3.ed.,New York,NY,USA: McGraw-Hill Book Company,1980

[6] Cook,R.D., Malkus; D.S.;Plesha,M.E. Concepts and Applications of Finite Element Analysis, 3. Ed..New York: John Wiley \& Sons, 1988.

[7] GERE, J. M., WEAVER JR., W., "Análisis de Estruturas Reticulares", Compañia Editorial Continental S.A., Mexico, 1976

[8] TIMOSHENKO, S.; YOUNG, D.H.; "Vibration Problems in Engineering", D. Van Nostrand Company, Inc.; New York, USA, 1955.

[9] WELLS, D. A.; "Theory and Problems of Lagrangian Dynamics", McGraw-Hill Book Company, New York, USA, 1967.

[10] DEN HARTOG, J. P., "Vibrações nos Sistemas Mecânicos", Editora da Universidade de São Paulo, São Paulo, SP, Brasil, 1972.

[11] THOMSON, W. T., "Teoria da Vibração com Aplicações”, Editora Interciência, Rio de Janeiro, RJ, Brasil, 1973.

\section{About Author (s):}

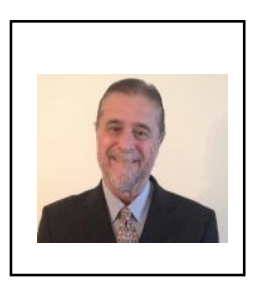

Avelino Alves Filho, Marine Engineer, MSc and PhD in Structural Analysis with Finite Element Method. General Director of NCE - www.nce.com.br in Brazil. Works with Structural Analysis for 40 years. Is author of 3 Books about Finite Element Method, about Linear Analysis, Dynamic Analysis and Nonlinear Analysis, and 11 books in Physics. Professor of Finite Element Method in Universities in Brazil.

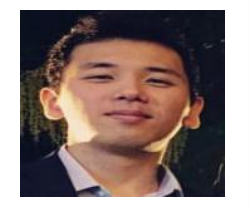

Lincoln Sadao Mazakina China, Mechanical Engineer, is developing a MSc Program in USP - São Paulo University State. Manager of R\&D Projects of NCE - www.nce.com.br in Brazil. Works with Finite Element Method. 